\title{
Clustering of parameter sensitivities: Examples from a helicopter airframe model updating exercise
}

\author{
H. Shahverdi ${ }^{\mathrm{a}}$, C. Mares ${ }^{\mathrm{b}}$, W. Wang ${ }^{\mathrm{a}}$ and J.E. Mottershead ${ }^{\mathrm{a}, *}$ \\ ${ }^{a}$ Mechanical Engineering Division, Department of Engineering, University of Liverpool, Liverpool, L69 3GH, UK \\ ${ }^{\mathrm{b}}$ School of Engineering and Design, Brunel University, Uxbridge, Middlesex, UB8 3PH, UK
}

Received 25 July 2007

Revised 27 January 2008

\begin{abstract}
The need for high fidelity models in the aerospace industry has become ever more important as increasingly stringent requirements on noise and vibration levels, reliability, maintenance costs etc. come into effect. In this paper, the results of a finite element model updating exercise on a Westland Lynx XZ649 helicopter are presented. For large and complex structures, such as a helicopter airframe, the finite element model represents the main tool for obtaining accurate models which could predict the sensitivities of responses to structural changes and optimisation of the vibration levels. In this study, the eigenvalue sensitivities with respect to Young's modulus and mass density are used in a detailed parameterisation of the structure. A new methodology is developed using an unsupervised learning technique based on similarity clustering of the columns of the sensitivity matrix. An assessment of model updating strategies is given and comparative results for the correction of vibration modes are discussed in detail. The role of the clustering technique in updating large-scale models is emphasised.
\end{abstract}

\section{Introduction}

The choice of parameters is one of the most important steps in updating a finite element model [1,2] and it is therefore worthwhile at the beginning to establish a few guiding principles. (i) The amount of information that can be obtained is limited and therefore taking more measurements in the same frequency range won't necessarily result in more information. Nor will the additional measurements necessarily allow more parameters to be estimated. (ii) The number of parameters should be considerably smaller than the number of measurements. The objective should be that the model-updating problem will be over-determined. Often the resulting equations are ill-conditioned and it is then necessary to apply additional information in the form of a side constraint by regularisation [3] - the reader is referred to the recent paper of Titurus and Friswell [4] for a qualitative treatment of Tikhonov regularisation in finite element model updating. (iii) The parameters should be justified by physical understanding of the structure under test and the test set-up. Ideally the chosen parameters should have a physical meaning directly, but this is not always possible in practice. Equivalent models and their parameters often lead to improved models when 'physical' parameters cannot be found. (iv) The data should be sensitive to small changes in the parameters. Parameterisation of finite element models for updating was considered in detail by Mottershead and his colleagues [5], including the use of equivalent models to represent the behaviour of mechanical joints. Link [6] used a two-part parameterisation to represent local uncertainties by physical parameters and global uncertainties by modal parameters of the residual structure.

\footnotetext{
*Corresponding author. E-mail: j.e.mottershead@liv.ac.uk.
} 


\section{Parameterisation of finite element models for updating}

Probably the simplest parameters for model updating are scalar multipliers applied at the element or substructure level. The updated model then takes the form,

$$
\begin{aligned}
& \mathbf{K}=\alpha_{1} \mathbf{K}_{1}+\alpha_{2} \mathbf{K}_{2}+\ldots+\alpha_{p} \mathbf{K}_{p} \\
& \mathbf{M}=\beta_{1} \mathbf{M}_{1}+\beta_{2} \mathbf{M}_{2}+\ldots+\beta_{q} \mathbf{M}_{q}
\end{aligned}
$$

where $p$ stiffness parameters and $q$ mass parameters are chosen for updating. Of course the same parameter $\alpha_{i}$ or $\beta_{j}$ may be applied to different elements, especially if they have very similar sensitivities. It would, of course, be undesirable to separately update elements with very similar sensitivities, a common cause of ill-conditioning in the updating equations.

The parameters $\alpha_{1}, \alpha_{2}, \ldots, \alpha_{p}$ and $\beta_{1}, \beta_{2}, \ldots, \beta_{q}$ should all begin the updating process with values of unity, thereby representing the initial finite element model. In this case the matrix derivatives, $\frac{\partial \mathbf{K}}{\partial \alpha_{i}}$ and $\frac{\partial \mathbf{M}}{\partial \beta_{j}}$ represent the $i^{\text {th }}$ element stiffness matrix, $\mathbf{K}_{i}$, and the $j^{\text {th }}$ element mass matrix, $\mathbf{M}_{j}$, respectively.

The 'automatic' selection of updating parameters may be carried out using methods based upon the ideas of subspace selection using the linearised sensitivity equation,

$$
\mathbf{S} \delta \theta=\delta \lambda
$$

where $\delta \lambda=\lambda_{m}-\lambda_{a}, \mathbf{S}$ denotes the matrix of sensitivities and $\delta \theta$ is the change in updating parameters to be determined. Clearly it is desirable to choose sensitive parameters, so that a small change has a significant effect in reducing the residual $\lambda_{m}-\lambda_{a}$, the difference between the measured data and an analytical prediction. The results depend upon the chosen linearisation point, i.e. the initial finite element model and its parameters, and also upon the scaling of Eq. (3). It is usual to apply scaling so that the prediction errors are divided by the prediction and the parameter errors are divided by the initial values of the parameters. Thus Eq. (3) may be expressed as,

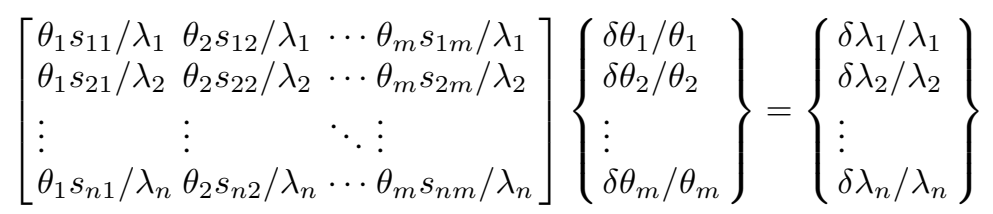

where $\mathbf{S} \in \Re^{n \times m}, \quad \delta \theta \in \Re^{m \times 1}, \quad \delta \lambda \in \Re^{n \times 1}, n>m$ for there to be a unique solution. In the above equations $\lambda$ is used to represent the data and is intended to be perfectly general so that natural frequencies, mode shapes and frequency response functions may all be included.

In practice a preliminary selection process takes place when deciding which parameters are to be entered in the vector $\delta \theta$. An important objective should be that $\operatorname{rank}(\mathbf{S})=m$, which means that parameters having a similar effect in the frequency range of interest should be clustered together to form a single updating parameter. Engineering judgement must be exercised in such cases. In complicated industrial structures such as the helicopter airframe considered in Section 6, parameters such as Young's Modulus or mass density are often chosen for updating. The changes in such parameters due to updating may not be physically meaningful but represent changes in stiffness or mass not readily available for correction (because of the extreme detail of the model) as equivalent parameters.

\section{Parameter subspace selection}

Methods for selecting the updated parameters are usually based on the 'cosine distance' between the $p^{\text {th }}$ column of $\mathbf{S}$ and the residual $\delta \lambda$.

$$
d_{p \lambda}=1-\cos \phi_{p \lambda}
$$

where $\phi_{p \lambda}$ is the angle between the vectors $\mathbf{s}_{p}$ and $\delta \lambda$. Equation (5) may be re-written as,

$$
d_{p \lambda}=1-\left(\mathbf{s}_{p}^{T} \delta \lambda\right) /\left(\left(\mathbf{s}_{p}^{T} \mathbf{s}_{p}\right)\left(\delta \lambda^{T} \delta \lambda\right)\right)^{1 / 2}
$$


having values between zero and unity. The best-subspace method [5] continues by finding the combination of two columns, $\mathbf{s}_{p}$ plus one other column $\mathbf{s}_{q}$, that best represents $\delta \lambda$ in the least-squares sense. At the $r^{\text {th }}$ step,

$$
\begin{gathered}
\min _{\mathbf{s}_{r}} \|\left[\left(\mathbf{I}-\left[\mathbf{s}_{p} \mathbf{s}_{q} \cdots \mathbf{s}_{r}\right]\left(\left(\begin{array}{c}
\mathbf{s}_{p}^{T} \\
\mathbf{s}_{q}^{T} \\
\vdots \\
\mathbf{s}_{r}^{T}
\end{array}\right)\left[\mathbf{s}_{p} \mathbf{s}_{q} \cdots \mathbf{s}_{r}\right]\right)^{-1}\left(\begin{array}{c}
\mathbf{s}_{p}^{T} \\
\mathbf{s}_{q}^{T} \\
\vdots \\
\mathbf{s}_{r}^{T}
\end{array}\right)\right] \delta \lambda \|_{2}\right. \\
\mathbf{s}_{p}, \mathbf{s}_{q}, \ldots, \mathbf{s}_{r} \in\left(\mathbf{s}_{1}, \mathbf{s}_{2}, \ldots, \mathbf{s}_{m}\right), \mathbf{s}_{r} \neq \mathbf{s}_{p}, \mathbf{s}_{q}
\end{gathered}
$$

results in the subspace $\left\{\mathbf{s}_{p} \mathbf{s}_{q} \cdots \mathbf{s}_{r}\right\}$ being defined and the parameters $\theta_{p}, \theta_{q}, \ldots, \theta_{r}$ selected successively.

An alternative, slightly more sophisticated approach is based on Gram-Schmidt orthogonalisation of the columns of $\mathbf{S}$. The method continues by orthogonalising $\delta \lambda$ and the remaining columns of $\mathbf{S}$ with respect to the chosen columns $\mathbf{s}_{p}, \mathbf{s}_{q}, \ldots, \mathbf{s}_{r}$ in turn. After the first step, defined in Eq. (6) the remaining columns of $\mathbf{S}$ are replaced by,

$$
\mathbf{s}_{i} \rightarrow \mathbf{s}_{i}-\mathbf{s}_{p}\left(\mathbf{s}_{p}^{T} \mathbf{s}_{i} / \mathbf{s}_{p}^{T} \mathbf{s}_{p}\right), \quad \mathbf{s}_{i} \neq \mathbf{s}_{p}, \quad i=1, \ldots, m
$$

where $\left(\mathbf{s}_{p}^{T} \mathbf{s}_{i} / \mathbf{s}_{p}^{T} \mathbf{s}_{p}\right)$ is the scalar multiplier that gives the projection of $\mathbf{s}_{i}$ on $\mathbf{s}_{p}$ and,

$$
\delta \lambda \rightarrow \delta \lambda-\mathbf{s}_{p}\left(\mathbf{s}_{p}^{T} \delta \lambda / \mathbf{s}_{p}^{T} \mathbf{s}_{p}\right)
$$

At this stage $\delta \lambda$ and all the remaining columns of $\mathbf{S}$ are confined to the space orthogonal to $\mathbf{s}_{p}$ and the parameter $\theta_{p}$ has been selected.

With the new $\mathbf{S}, \delta \lambda$ the search is begun again for the column $\mathbf{s}_{q}$ closest to $\delta \lambda$ that minimises the angle $\phi_{q}$ and therefore selects parameter $\theta_{q}$. The process is repeated until the required number of parameters, $r$, has been selected.

The Gram-Schmidt approach provides for a one-dimensional optimisation at each step. Both the Gram-Schmidt and best subspace methods are sub-optimal because at each step the previously selected parameters are retained. Also in both cases the selected parameters don't have to be sensitive ones and it is therefore best to either remove the insensitive parameters or to form clusters of parameters with columns deemed to be close to each other. In practice the columns of $\mathbf{S}$ may not be linearly independent, in which case after a number of iterations the remaining columns will be very similar to each other and no further progress can be made by continued iteration. A method is therefore needed for the identification of similar columns, especially for large-scale finite element models having a large number of candidate updating parameters.

In large-scale problems the comparison of the sensitivity matrix columns must be automated and clustering allows for a graphical interpretation of the results of such an exercise by the dendrogram diagram. In this paper we use clustering techniques for determining the groups of parameters having a similar effect on the variation of finite-element natural frequencies. Clustering methods are techniques that group together objects sharing the same property.

\section{Clustering of updating parameters}

The eigenvalue sensitivity matrix for a large finite element model will typically contain many quasi-dependent columns and to avoid this difficulty the subset of columns that are most independent may be chosen $[7,8]$ whilst the others are rejected. Clustering, which is described in detail by Everitt et al. [9], is one technique that can help the analyst ensure that the parameterisation represents the many different parts of the model by adding together the most similar columns of the sensitivity matrix. In this way the information available from the measurements is used to converge the model by the smallest adjustment to the updating parameters. In large-scale problems the comparison of the sensitivity matrix columns must be automated and clustering also allows for a graphical interpretation of the results of such an exercise by the clustering dendrogram.

To the authors' knowledge the clustering approach has not been applied before to the model updating problem, although it has been used in damage detection using modal data or acoustic emission data. Manson et al. [10] showed that three clusters were developed from the application of principal component analysis and Sammon 
mapping. Different physical behaviour in a cracked box girder was attributed to each of the three clusters. Within the development of specific structural monitoring health systems, clustering methods were integrated for improving the discrimination of structural conditions and to overcome the inherent uncertainties associated with the damage detection process [11-14]. A method involving a two-step procedure, for clustering of the data space into several regions and then applying principal component analysis in each local region was demonstrated in the monitoring a real bridge using in-situ vibration data $[15,16]$.

In the general application of the clustering method a distance criterion is needed to assess the similarity of different objects; the more similar two objects are, the closer they are and vice-versa. One feature of the recognition process involves the assessment of the relative distance between points, typically Euclidean, City block, Minkowski and Cosine distance [9]. The most commonly used distance measure is Euclidean distance, interpreted as physical distance between two $p$-dimensional points. The city block distance, equation (10b), describes distances on a rectilinear configuration. Both Euclidian $(r=2)$ and city block $(r=1)$ distances are special cases of the general Minkowski distance.

$$
d_{i j}=\left(\sum_{k=1}^{p}\left(x_{i k}-x_{j k}\right)^{2}\right)^{1 / 2} d_{i j}=\sum_{k=1}^{p}\left(x_{i k}-x_{j k}\right) \quad d_{i j}=\left(\sum_{k=1}^{p}\left(x_{i k}-x_{j k}\right)^{r}\right)^{1 / r} \quad(r \geqslant 1)
$$

$\begin{array}{lll}\text { a) Euclidean distance } & \text { b) City block distance } & \text { c) Minkowski distance }\end{array}$

When variables are measured on different scales, the difference between variable values is meaningless. In the cosine distance equation below, the cosine of the angle between two vectors $x_{i}$ and $x_{j}$ is subtracted from unity.

$$
\text { Cosine distance }: d_{i j}=1-\sum_{k=1}^{p} x_{i k} x_{j k} /\left(\sum_{k=1}^{p} x_{i k}^{2} \sum_{k=1}^{p} x_{j k}^{2}\right)^{1 / 2}
$$

For example the three-dimensional points $x_{i}=(1,2,3)$ and $x_{j}=(3,6,9)$ have $d_{i j}=0$ (based on the cosine distance) while $x_{j}$ is three times the size of $x_{i}$.

After defining the similarity criterion, it is necessary to decide upon the method of linking the similar objects within the cluster. A hierarchical dendrogram can be defined using any of the following methods:

Single: Shortest distance

Complete: The furthest distance

Average: Unweighted Pair Group Method with Arithmetic Mean (UPGMA)

Weighted: Weighted Pair Group Method with Arithmetic Mean (WPGMA)

Centroid: Unweighted Pair-Group Method using Centroids (UPGMC)

Median: Weighted Pair-Group Method using Centroids (WPGMC)

One of the simplest hierarchical clustering methods is the single linkage. In this method the distance between groups is defined as that of the closest pair of individuals. The complete linkage is opposite to single linkage and defined as the furthest pair of individuals. Both methods require a proximity matrix and do not require access to a data matrix. Average linkage (UPGMA), uses the average distance between all pairs of objects in two clusters. Weighted (WPGMA) linkage is similar to group average linkage but weights inter-cluster distances according to the inverse of the number of objects in each class. Another agglomerative hierarchical method is the centroid also known as UPGMC, which uses the data matrix rather than a proximity matrix and merges the clusters with the most similar mean vector. The output in this method is only meaningful if the distance calculated is based on Euclidian distances. Median linkage (WPGMC) is similar except that the centroids of merging clusters are weighted equally to produce the new centroid of the merged cluster. Figure 1 shows how different linkage methods work according to the single, the complete, the average and the centroid definitions.

Amongst different hierarchical clustering schemes the Un-weighted Pair Group Method with Arithmetic Mean (UPGMA) was used in the present study. This method of clustering measures the average distance between a pair of objects. It is relatively robust and takes account of the cluster structure [7]. The single and the complete linkage methods do not take account of cluster structure. Other linkage methods are based on the Euclidian distance, which is not applicable in the present case.

An example is shown in Fig. 2 to clarify how hierarchical clustering works. Seven objects are shown in the XY plane and the Euclidian distance to measure the similarity between each object and the single method of linking the closest two objects are used. Further explanation and examples may be found in [7]. 


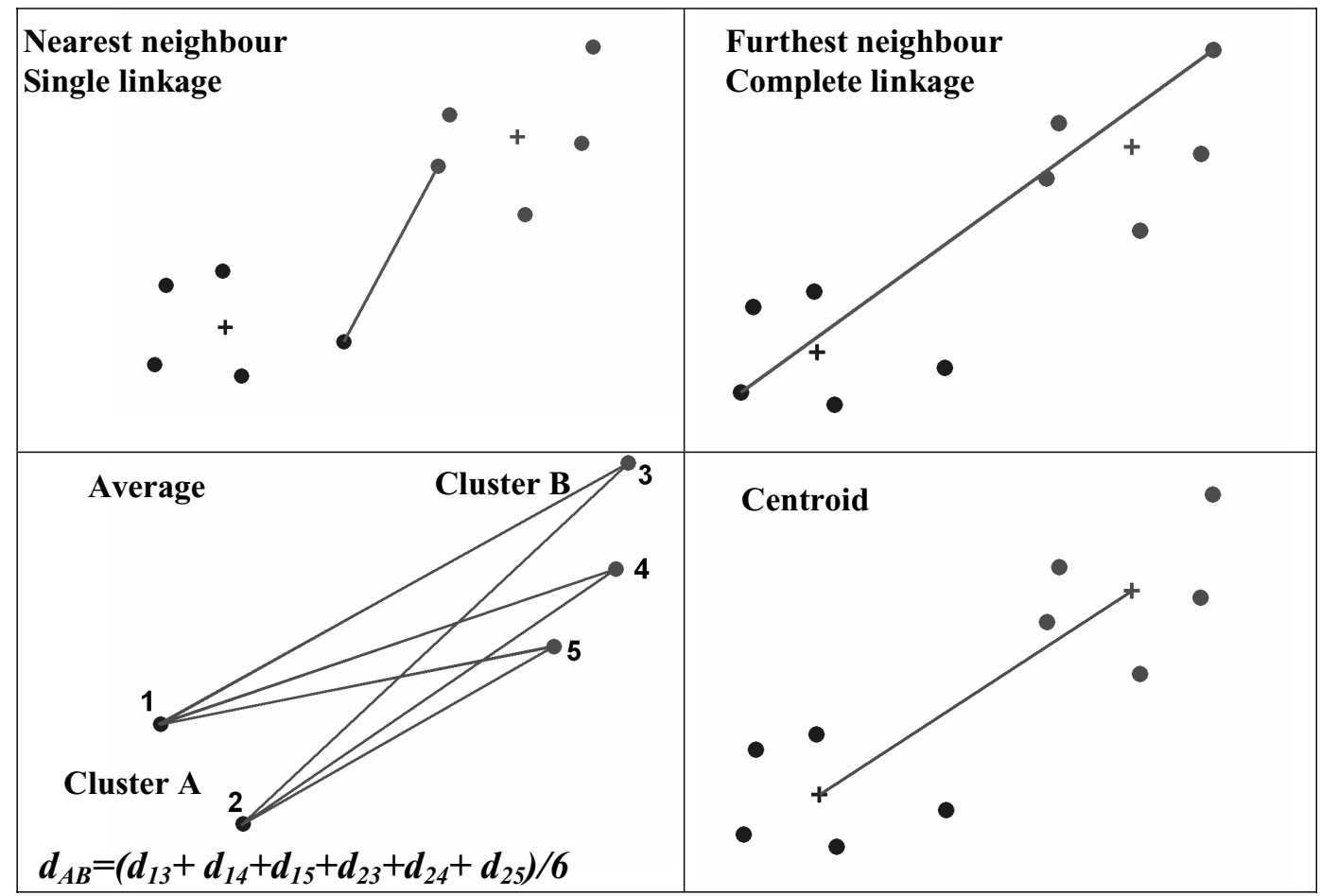

Fig. 1. Different clustering schemes (The + signs mark the centres of the two clusters).

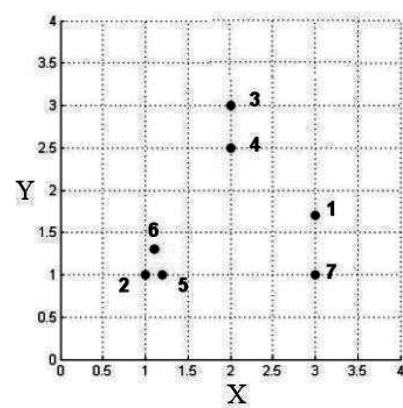

\begin{tabular}{|c|c|c|}
\hline objects & $\mathbf{X}$ & $\mathbf{Y}$ \\
\hline 1 & 3 & 1.7 \\
\hline 2 & 1 & 1 \\
\hline 3 & 2 & 3 \\
\hline 4 & 2 & 2.5 \\
\hline 5 & 1.2 & 1 \\
\hline 6 & 1.1 & 1.3 \\
\hline 7 & 3 & 1 \\
\hline
\end{tabular}

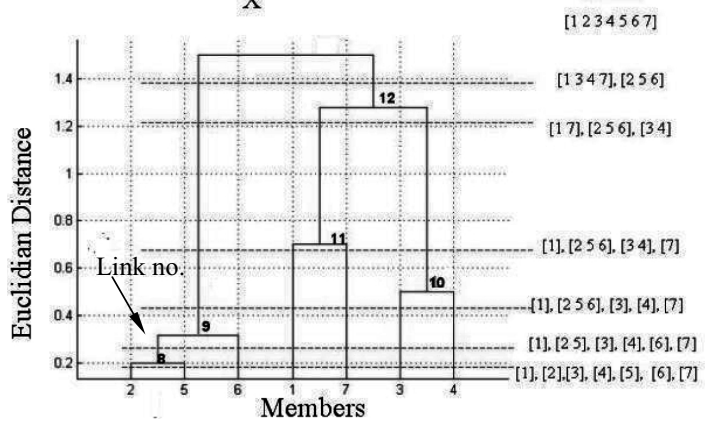

\begin{tabular}{|c|c|c|c|}
\hline $\begin{array}{c}\text { Link } \\
\text { no. }\end{array}$ & \multicolumn{2}{|c|}{$\begin{array}{c}\text { linked } \\
\text { objects }\end{array}$} & distance \\
\hline 8 & 2 & 5 & 0.2 \\
\hline 9 & 6 & 8 & 0.32 \\
\hline 10 & 3 & 4 & 0.5 \\
\hline 11 & 1 & 7 & 0.7 \\
\hline 12 & 10 & 11 & 1.28 \\
\hline & 9 & 12 & 1.5 \\
\hline
\end{tabular}

Fig. 2. Hierarchical clustering, single linkage.

\section{Example 1: Helicopter tail cone}

In the first example the tailcone finite element model of a Westland Lynx XZ649 helicopter was updated using measured natural frequencies. The tailcone was attached to a rigid wall and 57 accelerometer measurements were 
Table 1

Comparisons of analytical and predicted natural frequencies $(\mathrm{Hz})$ and errors (\%)

\begin{tabular}{clrcrrr}
\hline Mode no. & Mode description & $\begin{array}{c}\text { Test } \\
(\mathrm{Hz})\end{array}$ & $\begin{array}{c}\text { Initial } \\
\text { FEM }(\mathrm{Hz})\end{array}$ & $\begin{array}{c}\text { Error } \\
(\%)\end{array}$ & $\begin{array}{r}\text { Updated } \\
\text { FEM }(\mathrm{Hz})\end{array}$ & \multicolumn{1}{c}{$\begin{array}{c}\text { Error } \\
(\%)\end{array}$} \\
\hline 1 & 1st lateral + vertical bending & 12.66 & 15.46 & 22.12 & 12.86 & 1.58 \\
2 & 1st vertical + lateral bending & 13.22 & 16.09 & 21.71 & 13 & -1.66 \\
3 & 2nd lateral bending & 45.90 & 51.70 & 12.64 & 46.52 & 1.35 \\
4 & 2nd vertical bending & 58.12 & 68.90 & 18.55 & 58.09 & -0.05 \\
5 & 3rd lateral Bending & 118.13 & 130.25 & 10.26 & 117.18 & -0.80 \\
6 & Tail pylon (fore\& aft) & 122.09 & 109.87 & -10.01 & 108.26 & -11.33 \\
7 & Tail pylon (rotation) & 150.19 & 156.52 & 4.21 & 147.14 & -2.03 \\
\hline
\end{tabular}

Table 2

Predicted natural frequencies $(\mathrm{Hz})$ and errors $(\%)$ between the finite element model (base line) and test data: Initial model

\begin{tabular}{clccc}
\hline Mode no. & Mode description & Initial FEM $(\mathrm{Hz})$ & Test $(\mathrm{Hz})$ & Error $(\%)$ \\
\hline 1 & 1st vertical bending & 13.30 & 12.45 & 7.01 \\
2 & 1st lateral bending & 14.00 & 13.29 & 5.13 \\
3 & Tail plane fore/aft bending & 19.60 & 18.52 & 6.06 \\
4 & Tail plane vertical bending & 22.80 & 19.85 & 15.10 \\
5 & 2nd vertical Bending & 35.30 & 32.67 & 7.97 \\
6 & 2nd lateral Bending & 38.50 & 36.91 & 4.38 \\
7 & Fuselage torsion & 47.10 & 50.34 & -6.50 \\
8 & 3rd vertical bending & 56.30 & 52.57 & 7.19 \\
& & - & 54.97 & \\
9 & 3rd lateral bending & 64.00 & 64.67 & -1.02 \\
10 & Cabin roof bending & 71.10 & 64.69 & 9.97 \\
11 & Cabin roof bending & 71.40 & - & - \\
12 & Cabin floor vertical bending & 75.00 & 62.59 & -19.80 \\
\hline
\end{tabular}

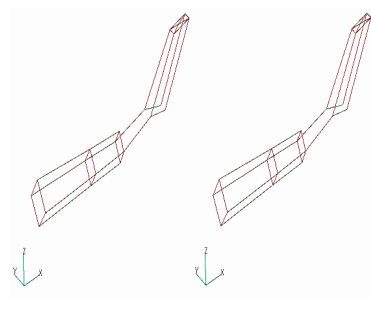

(a)

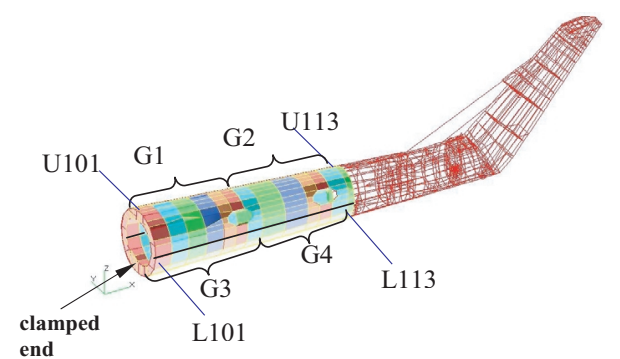

(b)

Fig. 3. a) the tailcone test grid points; b) FE model with 13 subgroups on upper and lower halves.

taken in the frequency range $0-200 \mathrm{~Hz}$. Owing to the closely symmetric structure of the tailcone, pairs of close modes were present in the low frequency range and 8 modes of vibration were extracted up to $156 \mathrm{~Hz}$.

An eigenvalue sensitivity study was carried out using plate thicknesses for the tailcone. To study the finite element model in detail, the tail cone upper and lower skin which was divided into 13-sections closest to the wall, using different colours as shown in Fig. 3 (colours are shown on the electronic version available from the journal website). The section indices were U101-U113, representing the upper-half of the tailcone and L101-L113 representing the lower half; the sensitivities of the upper and lower halves has been to be quite different possibly due to stiffening by added plates etc. Based on the result shown in Fig. 4 it was decided that the tailcone parameters could be clustered into four groups: G1, G2, G3 and G4. The hierarchical clustering (UPGMA) diagram shown in Fig. 4 uses a cosine distance to represent the closeness of the columns of the sensitivity matrix.

Model updating was carried out using only four parameters for the tail upper and lower parts as explained together with stiffness of the springs modelled to represent the joint between the tail and the wall, resulting in considerable improvement in the first seven tail vibration modes, as shown in Table 1. 


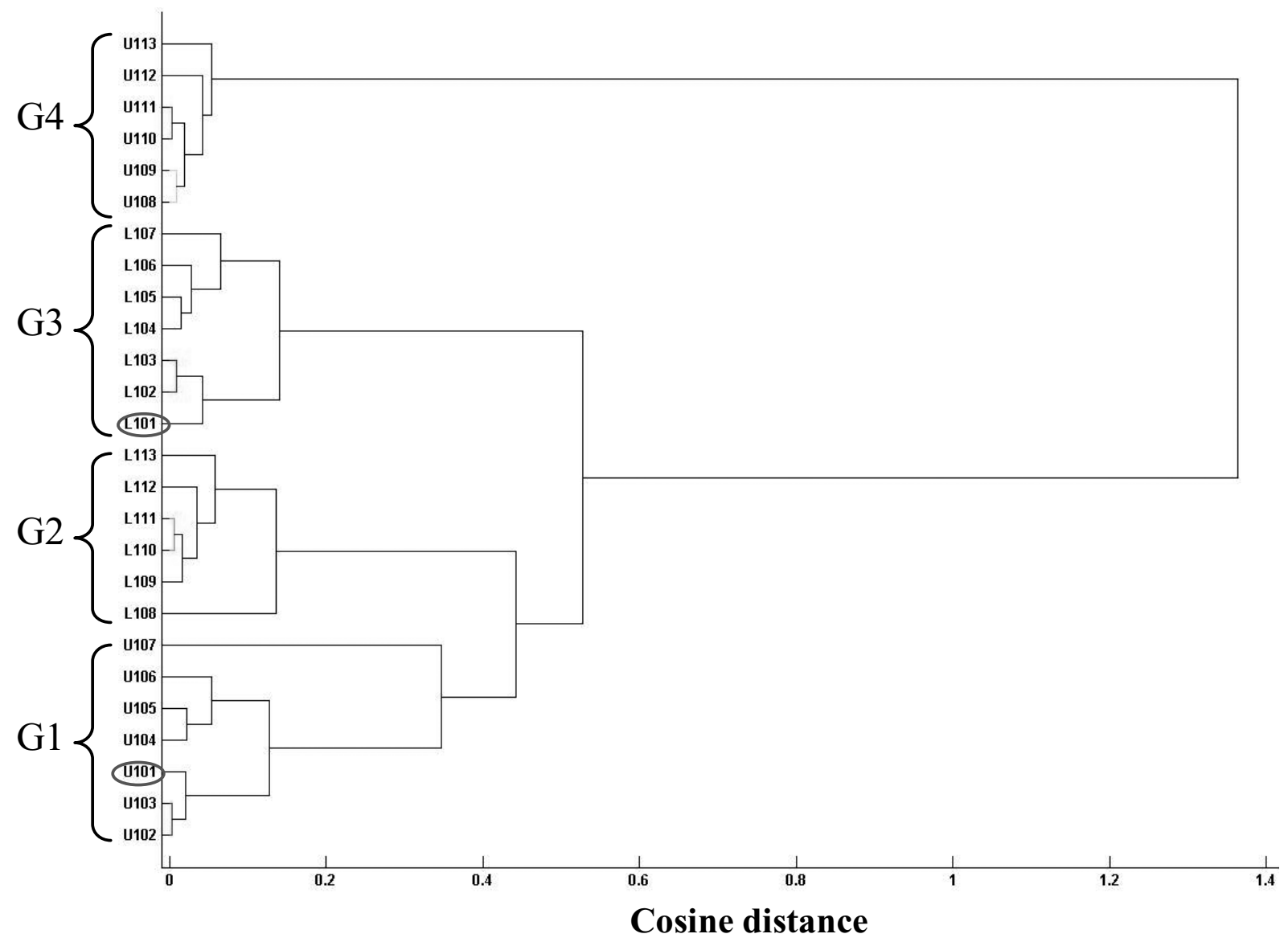

Fig. 4. Hierarchical clustering (UPGMA) of the 26 columns of sensitivity results of the tail sections (upper and lower halves).

\section{Example 2: Helicopter air frame}

Initially 109 different finite-element material properties were used to define the whole helicopter model. It was observed that ten material properties, spread over different parts of the model, were most important. The model was partitioned to form smaller groups, thereby defining a set of parameters based on engineering judgement as well as topological and technical considerations. Then an eigenvalue sensitivity study for the whole helicopter model was carried out using the elastic moduli for 147 groups of elements. The first 36 columns of the scaled sensitivity matrix (the most sensitive parameters) are shown in Fig. 5. In the horizonal plane of the figure the parameter indices are shown against the first eight natural frequencies. The height of the columns denoted the scaled sensitivity of each natural frequency to each of the 36 elastic moduli, the updating parameters. The sensitivities are all positive since an increase in stiffness generally results in an increase in all the natural frequencies.

Clustering was the principal method employed to finally reduce the updating parameters to an acceptable number. The dendrogram of the cabin parameters provides excellent insight for the selection of updating parameters using the cosine distance between three groups as shown in Fig. 6.

Vibration tests were carried out on the baseline configuration, without rotors and gearboxes, in free-free conditions. Doors, drive shafts and all removable panels were removed. Mechanical linkages in the cabin area were fixed in order to prevent them from affecting the modal test. Five shakers were used to excite the structure. Four excitation points were located on the nose and tail in $\mathrm{Y}$ and $\mathrm{Z}$ directions and one excitation point was under the cabin frame in the $\mathrm{X}$ direction. Eighty-seven measurements points were used at 29 different positions as shown in Fig. 7. The frequency range of the modal test was $0-104 \mathrm{~Hz}$, the analysis being carried out in the frequency domain. Twelve modes of vibration (up to $75 \mathrm{~Hz}$ ) were extracted from the baseline-structure test. 
Table 3

Predicted natural frequencies $(\mathrm{Hz})$ and errors $(\%)$ between the finite element model (base line) and test data: After correcting the spar offset

\begin{tabular}{clccc}
\hline Mode no. & Mode description & Modified FEM $(\mathrm{Hz})$ & Test $(\mathrm{Hz})$ & Error $(\%)$ \\
\hline 1 & 1st vertical bending & 13.25 & 12.45 & 6.39 \\
2 & 1st lateral bending & 14.07 & 13.29 & 5.93 \\
3 & Tail plane fore/aft bending & 20.03 & 18.52 & 8.15 \\
4 & Tail plane vertical bending & 21.57 & 19.85 & 8.66 \\
5 & 2nd vertical Bending & 36.62 & 32.67 & 12.11 \\
6 & 2nd lateral Bending & 38.08 & 36.91 & 3.18 \\
7 & Fuselage torsion & 49.11 & 50.34 & -2.44 \\
8 & 3rd vertical bending & 57.74 & 52.57 & 9.85 \\
& & & 54.97 & \\
9 & 3rd lateral bending & 67.78 & 64.67 & 4.82 \\
10 & Cabin roof bending & 71.99 & 64.69 & 11.29 \\
11 & Cabin roof bending & 74.55 & - & \\
12 & Cabin floor vertical bending & 75.52 & 62.59 & 20.66 \\
\hline
\end{tabular}

Mode no.

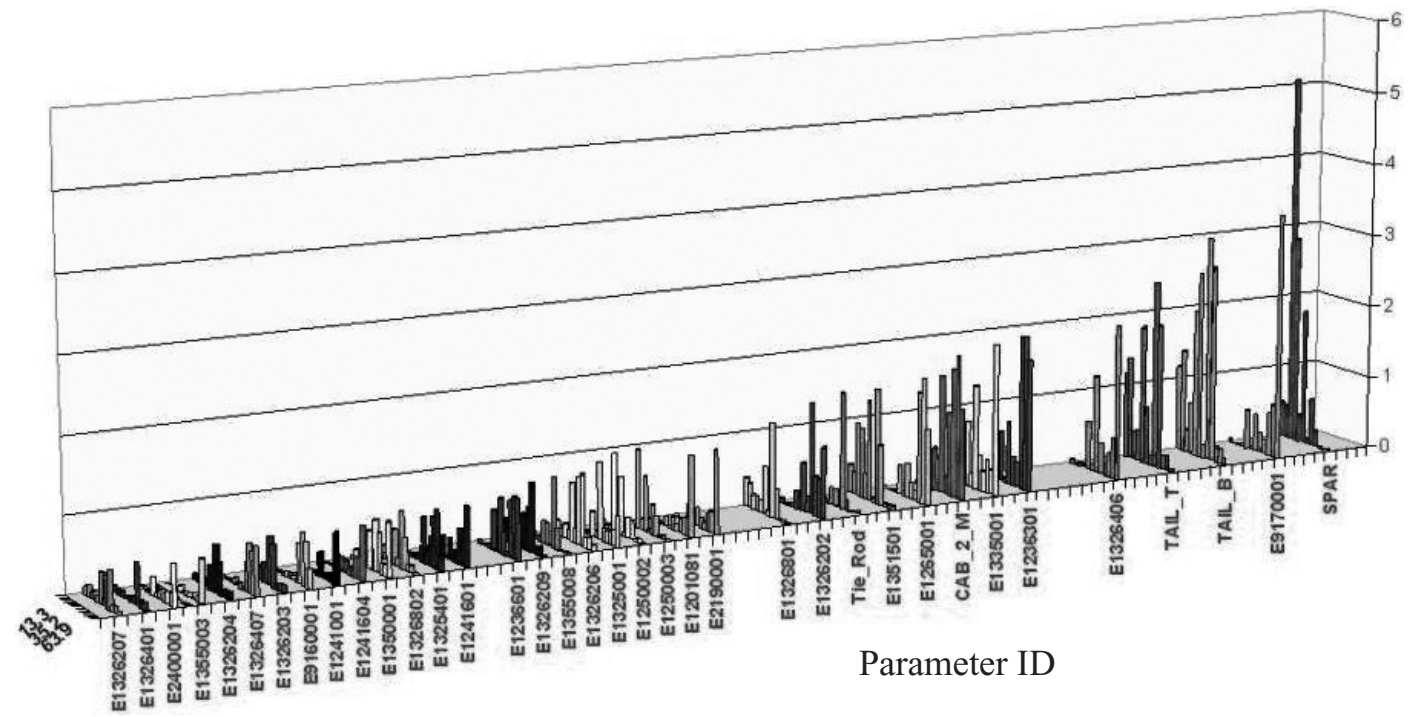

Fig. 5. Eigenvalue sensitivity to Young's modulus: 36 most important groups.

Figure 8 shows a comparison between the finite-element predicted modes and the test modes. Table 2 shows that the natural frequencies predicted by the analytical model (bending modes) are higher than the test results and amongst them; the vertical ones are more in error than the lateral ones. As mentioned previously the tail has a quasi-symmetric structure and the tail mostly dominates the early modes, which appear in pairs. The tailcone shows more flexibility in the test than the model, due to its riveted multi layer skin.

The tail spar was one of the most sensitive areas of the model and close inspection revealed that it had been incorrectly modelled. After correction, by adding an offset the FE-test correlation was significantly improved. Although the modification reduced the error for mode 4 to $8 \%$ it was not successful in improving mode 3 as can be seen in Table 3.

Using subset selection in conjunction with hierarchical clustering techniques (UPGMA), helped in finding suitable parameters and the updated model showed major improvements in terms of MAC and frequency agreement. The average frequency error was reduced from $6.6 \%$ to $2 \%$ and the MAC was improved too. The final results obtained after model updating are presented in the Table 4 and Fig. 9. The chosen updating parameters together with correction factors are shown in Table 5 and Fig. 10. It should be recalled that the chosen elastic moduli are equivalent parameters representing changes to the stiffness of the structure not readily available for correction directly because of the extreme detail present in the large finite element model. 
Table 4

Predicted natural frequencies $(\mathrm{Hz})$ and errors $(\%)$ between the finite element model (base line) and test data: After updating

\begin{tabular}{clccc}
\hline Mode no. & Mode description & Updated FEM $(\mathrm{Hz})$ & Test $(\mathrm{Hz})$ & Error $(\%)$ \\
\hline 1 & 1st vertical bending & 12.06 & 12.45 & -3.12 \\
2 & 1st lateral bending & 13.14 & 13.29 & -1.08 \\
3 & Tail plane fore/aft bending & 18.41 & 18.52 & -0.61 \\
4 & Tail plane vertical bending & 19.94 & 19.85 & 0.47 \\
5 & 2nd vertical Bending & 34.15 & 32.67 & 4.55 \\
6 & 2nd lateral Bending & 36.83 & 36.91 & -0.20 \\
7 & Fuselage torsion & 48.25 & 50.34 & -4.16 \\
8 & 3rd vertical bending & 54.33 & 52.57 & 3.36 \\
& & 62.97 & 54.97 & - \\
9 & 3rd lateral bending & 63.95 & 64.67 & - \\
10 & Cabin roof bending & 67.31 & 64.69 & - \\
11 & Cabin roof bending & 71.48 & - & - \\
12 & Cabin floor vertical bending & 72.77 & 62.59 & - \\
\hline
\end{tabular}

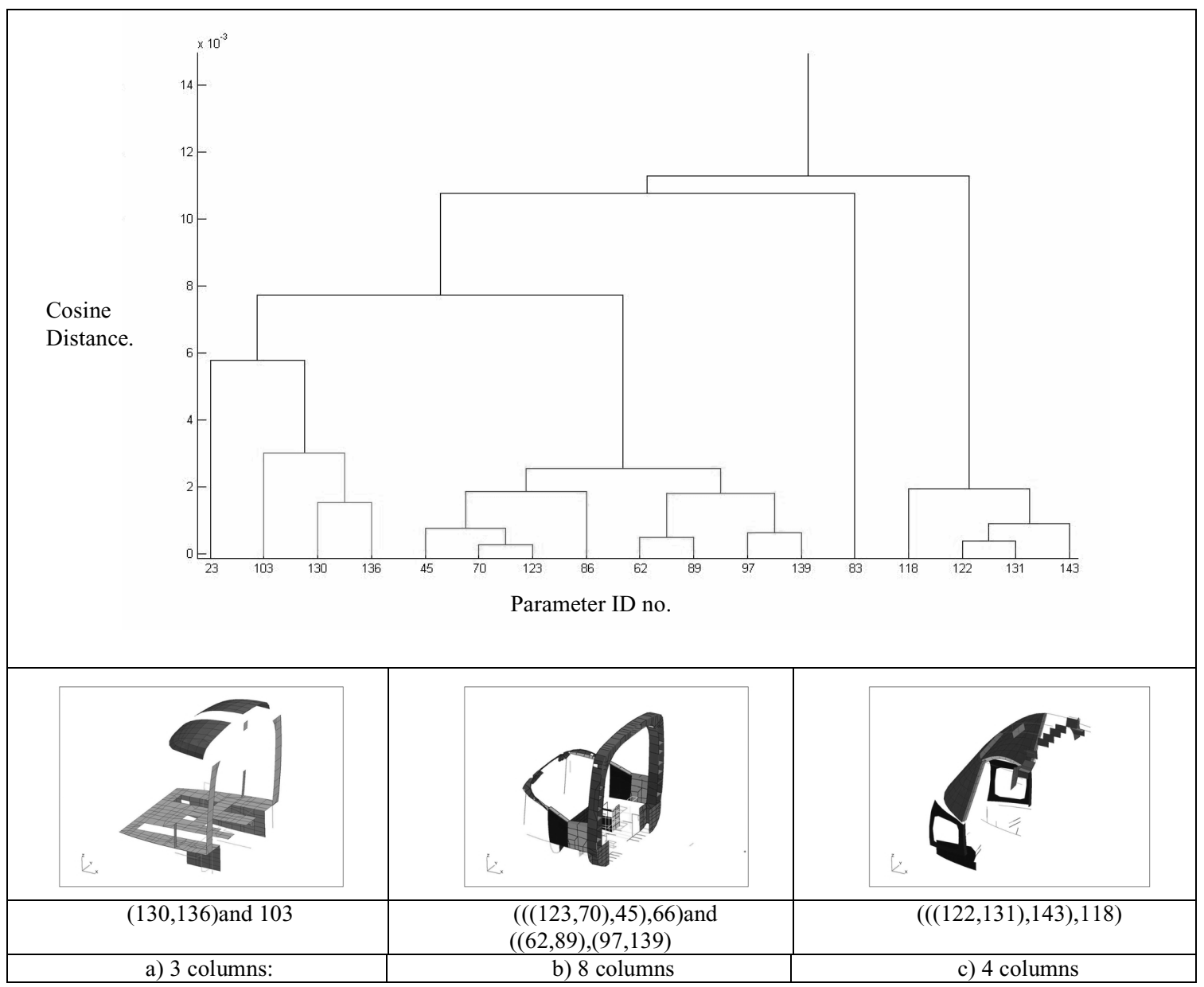

Fig. 6. Clustering of three groups in the cabin region: $5^{\circ}$ angle criterion.

Parameter E1312001 represents the upper half of the tailcone, which is riveted, has many patches and open access points that might lead to a stiffness reduction represented by a reduced Young's modulus in the updated parameters. Another possibility is that the added patches would lead to an increase in mass having the same effect as a stiffness 
Table 5

The selected parameters and correction factors for Young's modulus

\begin{tabular}{ccc}
\hline & Parameter & Correction factor \\
\hline 1 & E1312001 & $4.67 \times 10^{-1}$ \\
2 & E1326203 & $6.42 \times 10^{-1}$ \\
3 & E1326209 & $8.78 \times 10^{-1}$ \\
4 & E1355010 & $8.44 \times 10^{-1}$ \\
5 & E1250000 & $4.16 \times 10^{-1}$ \\
6 & E1250001 & $8.43 \times 10^{-1}$ \\
7 & E1201081 & $4.44 \times 10^{-1}$ \\
8 & E2190001 & $4.44 \times 10^{-1}$ \\
9 & E9170001 & $4.44 \times 10^{-1}$ \\
\hline
\end{tabular}

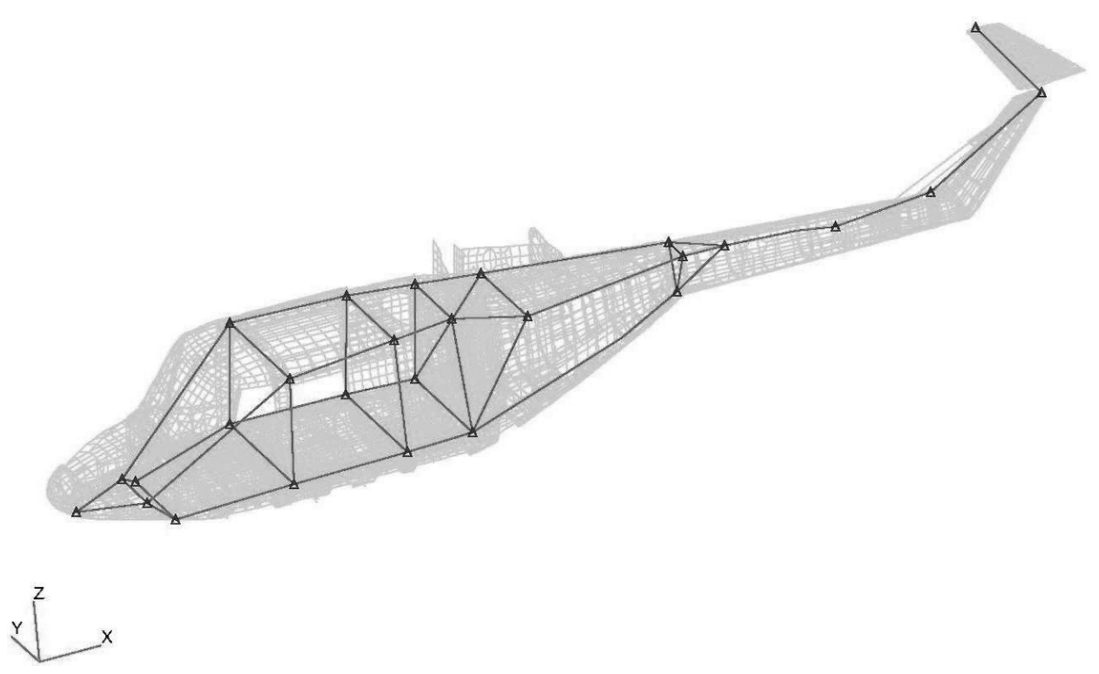

Fig. 7. Finite element model of the helicopter with test wire frame.

reduction.

Regions of connectivity between the tapered section of the cabin and tail-cone, and the tapered section and the cabin itself are represented by parameters E1326203 and E1326209. The results suggest that these physical transitions are less stiff than in the model.

The region is at the elbow of the tail-cone and at one end of the tie rods coincides with parameter E1335010. Again a stiffness reduction is indicated.

Parameters E1250000 and E1250001 represent the tail spar and tail plane. The tail spar has been modelled using a single beam element. Over-stiffening of the joint in the FE model is the likely cause of the discrepancy.

The three groups E1201081, E2190001 and E9170001 were linked together to form a single updating parameter affecting mainly the third vertical bending mode. The boundary condition of the windshield with the cabin structure may be responsible for the indicated stiffness reduction.

\section{Conclusions}

The parameter clustering method is demonstrated in the sensitivity analysis of a Westland Lynx XZ649 helicopter and model updating of the tailcone. The clustering method is useful in developing a good engineering understanding of the model and the test structure. It enables those parameters having a similar effect to be recognised and included together in a small number of powerful clusters of parameters for model updating.

Clustering methods can be used to improve the condition of the sensitivity matrix during model updating. At the same time, their graphical representation gives a better insight into the model behaviour. However, if the columns of the sensitivity matrix change during the updating process, then it is possible that different clusters should be formed. 

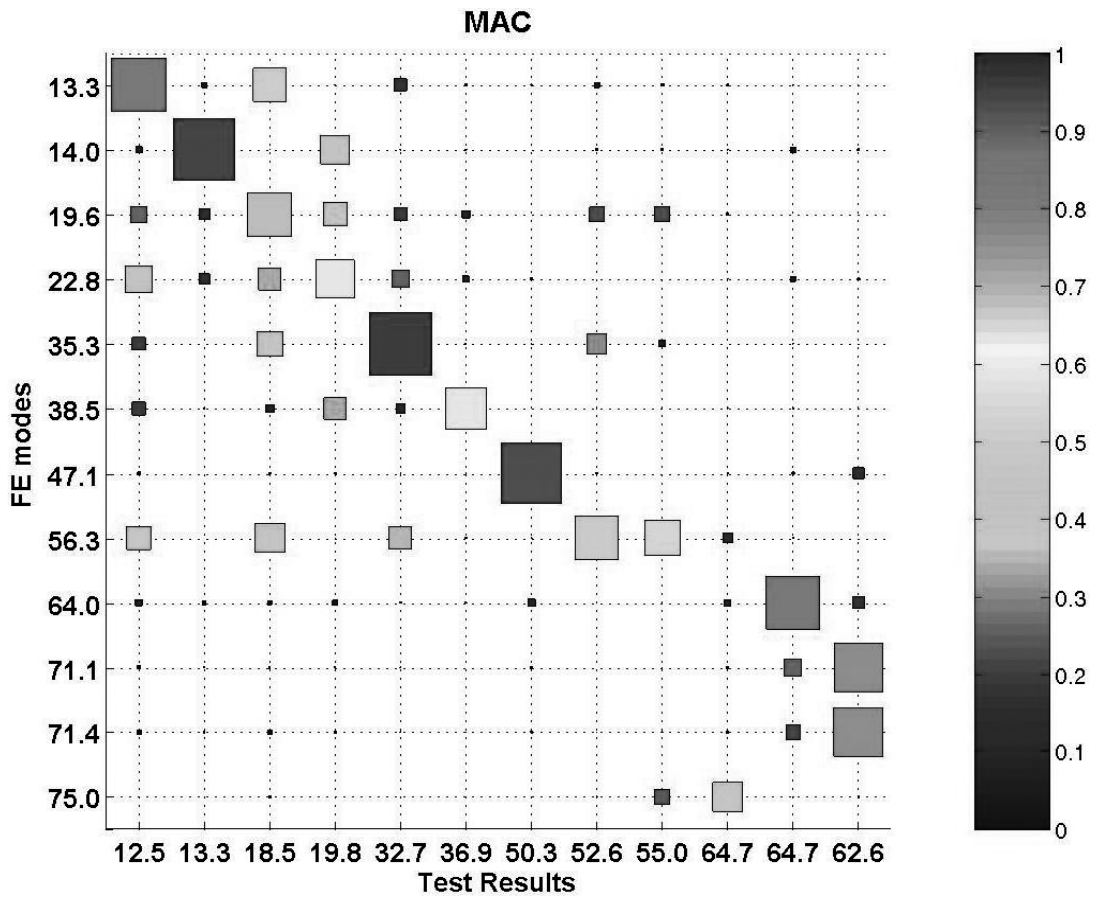

Fig. 8. Correlation of the finite element model and test data.

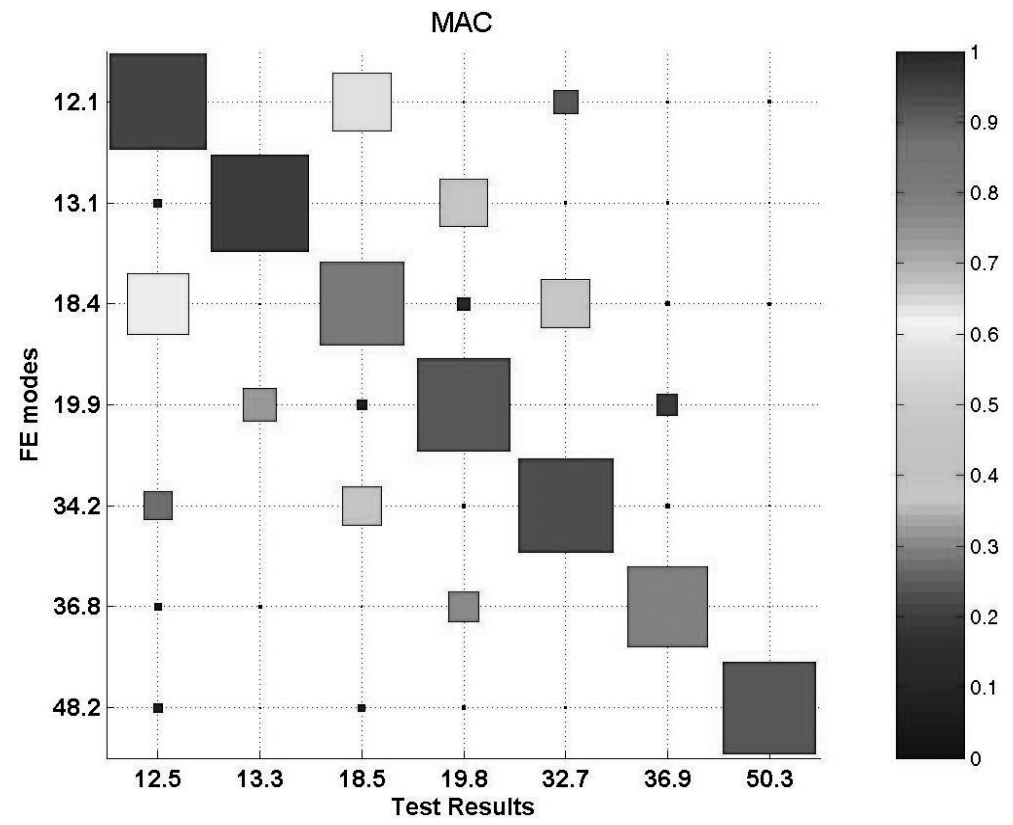

Fig. 9. MAC table after offset correction and updating.

It is known that there are principally two tools, sensitivity analysis and subset selection, for the selection of parameters in model updating. In the case of very large and complicated systems these two tools on their own are insufficient to compare the many possible sensitivity columns. Clustering methods are able to carry out this task efficiently and together with sensitivity analysis and subset selection provide a method for including the effects of 


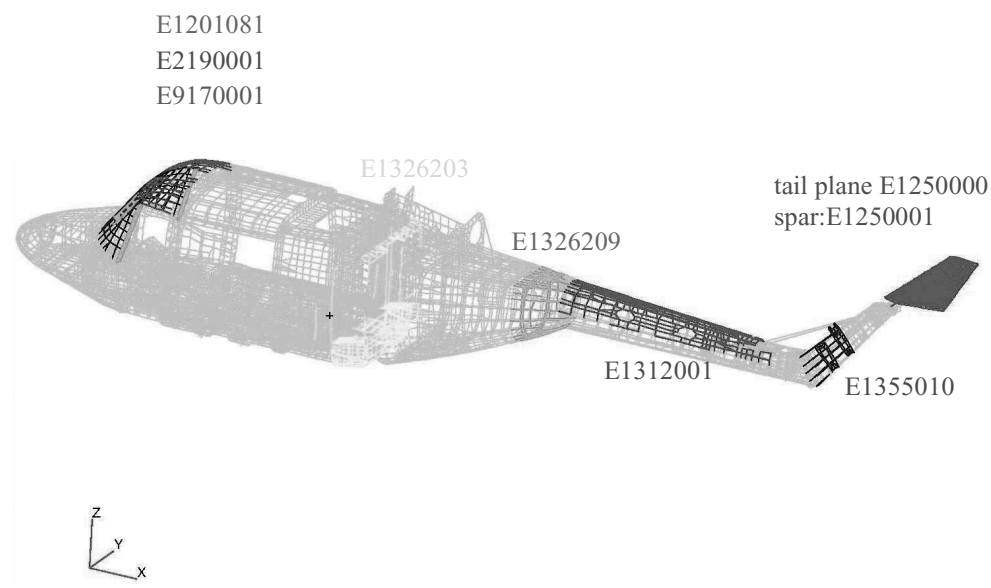

Fig. 10. Selected updating parameters.

changes to less sensitive substructures by combining those columns of the sensitivity matrix that are close (within a tolerance band) over the range of frequencies of the test.

\section{Acknowledgement}

The authors wish to thank QinetiQ Ltd for supplying the finite element model, the test results, and for permission to publish the results of the research described in this paper. The research formed part of a collaborative programme carried out under GARTEUR AG14.

\section{References}

[1] M.I. Friswell and J.E. Mottershead, Finite Element Model Updating in Structural Dynamics, Kluwer Academic Publishers, London, 1995.

[2] J.E. Mottershead and M.I. Friswell, Model updating in structural dynamics: a survey, Journal of Sound and Vibration 167(2) (1993), 347-375.

[3] H. Ahmadian, J.E. Mottershead and M.I. Friswell, Regularisation methods for finite element model updating, Mechanical Systems and Signal Processing 12(1) (1998), 47-64.

[4] B. Titurus and M.I. Friswell, Regularisation in model updating, International Journal for Numerical Methods in Engineering, in press.

[5] J.E. Mottershead, C. Mares, M.I. Friswell and S. James, Selection and updating of parameters for an aluminium space-frame model, Mechanical Systems and Signal Processing 14(6) (2000), 923-944.

[6] M. Link, Updating analytical models by using local and global parameters and relaxed optimisation requirements, Mechanical Systems and Signal Processing 12(1) (1998), 7-22.

[7] G. Lallement and J. Piranda, Localization methods for parameter updating of finite element models in elastodynamics, 8th International Modal Analysis Conference, Florida, Orlando, 1990, 579-585

[8] M.I. Friswell, J.E. Mottershead and H. Ahmadian, Combining subset selection and parameter constraints in model updating, Trans ASME, $J$ Vibration and Acoustics 120 (1998), 854-859.

[9] B.S. Everitt, S. Landau and M. Leese, Cluster Analysis, London, 2001.

[10] G. Manson, K. Worden, K. Holford and R. Pullin, Visualisation and dimension reduction of acoustic emission data for damage detection, J Intelligent Material Systems and Structures 12 (2001), 529-536.

[11] G. Garcia, K. Butler and N. Stubbs, Relative performance of clustering-based neural network and statistical pattern recognition models for non-destructive damage detection, Smart Materials and Structures 6(4) (1997), 415-424.

[12] L. Meyyappan and C. Dagli, Fuzzy-neuro system for bridge health monitoring, Intelligent Engineering Systems Through Artificial Neural Networks 13 (2003), 565-570.

[13] A. Lazarevic, R. Kanapadya, K. Tamma, C. Kamath and V. Kumar, Damage Prediction in Structural Mechanics Using Partitioning Approach, in: Data Mining and Knowledge Discovery: Theory, Tools, V. Technology and Belur V. Dasarathy, eds, Proceedings of SPIE Vol. 5098, 2003.

[14] G. Mustafa, F. Necati Catbasa and M. Georgiopoulos, Application of Pattern Recognition Techniques to Identify Structural Change in a Laboratory Specimen, in: Sensors and Smart Structures Technologies for Civil, Mechanical, and Aerospace Systems, Masayoshi Tomizuka, Chung-Bang Yun and Victor Giurgiutiu, eds, Proc. of SPIE Vol. 6529, 2007. 
[15] A.-M. Yan, G. Kerschen, P. De Boe and J.-C. Golinval, Structural damage diagnosis under varying environmental conditions - Part I: A linear analysis, Mechanical Systems and Signal Processing 19 (2005), 847-864.

[16] A.-M. Yan, G. Kerschen, P. De Boe and J.-C. Golinval, Structural damage diagnosis under varying environmental conditions - part II: local PCA for non-linear cases, Mechanical Systems and Signal Processing 19 (2005), 865-880. 

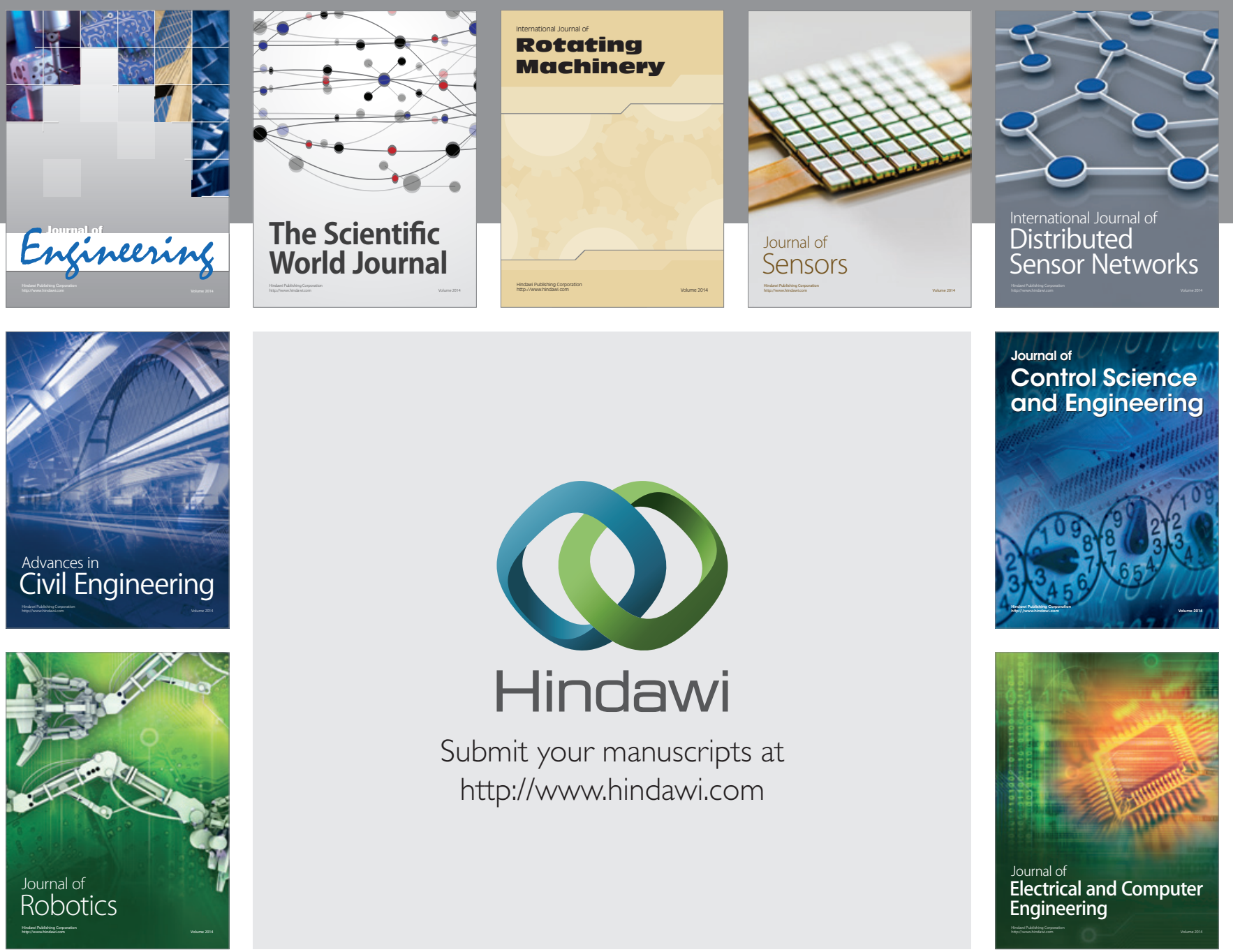

Submit your manuscripts at

http://www.hindawi.com
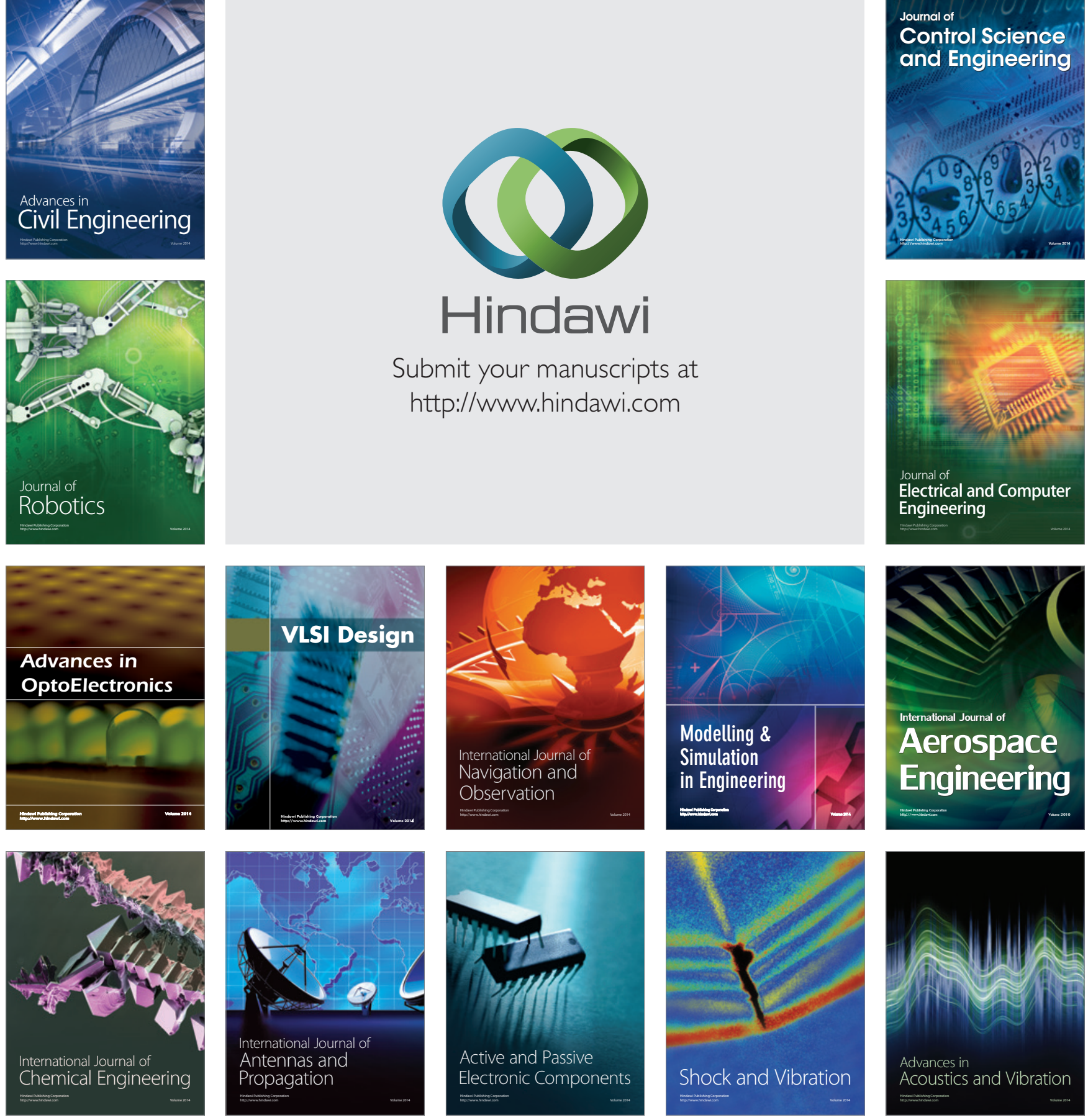